\title{
Miocardiopatía por estrés: análisis de 17 casos
}

\section{Stress cardiomyopathy: analysis of 17 cases}

Pablo Young', Anyella Dallanna Posada Guzmán', Débora Pellegrini', Bárbara C. Finn', Osvaldo Manuale², Pablo Sorensen², Javier Barcos², José Gargiulo², Glenda Ernst ${ }^{3}$, Julio E. Bruetman'

\section{RESUMEN}

Introducción. La miocardiopatía por estrés (MCS) o síndrome de takotsubo, se caracteriza por una disfunción sistólica aguda y transitoria que simula la presentación de un síndrome coronario agudo gatillada por un factor estresante psicológico o físico.

Métodos. Se presenta una serie de casos de pacientes con MCS del Hospital Británico entre 201 I y 2016 . Se describen las características de la población estudiada y factores epidemiológicos y clínicos asociados.

Resultados. Se incluyeron 17 pacientes con una edad media de 62,5 años, de los cuales el $94 \%$ eran mujeres. Más del $70 \%$ presentaban uno o más factores de riesgo cardiovascular. La mayoría tenían valores elevados de los biomarcadores miocárdicos troponina T ultrasensible y creatinfosfokinasa. Se observó compromiso apical y medioventricular del ventrículo izquierdo con afectación moderada a severa de la fracción de eyección del ventrículo izquierdo e insuficiencia mitral variable. La evolución de la mayoría de los pacientes fue favorable.

Discusión. La incidencia de este síndrome ha ido en aumento en la última década, debido al mayor conocimiento de la enfermedad y a los avances y disponibilidad de los medios de diagnóstico por imágenes. Los hallazgos de esta serie podrían contribuir a identificar rápidamente a los pacientes con miocardiopatía por estrés y optimizar su tratamiento y manejo clínico.

Palabras clave: miocardiopatía de takotsubo; miocardiopatía por estrés; síndrome de corazón roto; síndrome de abombamiento apical, síndrome de aturdimiento apical transitorio del ventrículo izquierdo.

Palabras clave: miocardiopatía de takotsubo, miocardiopatía por estrés, síndrome de corazón roto, síndrome de abombamiento apical, síndrome de aturdimiento apical transitorio del ventrículo izquierdo.

\section{ABSTRACT}

Introduction. Stress cardiomyopathy (SCM) or takotsubo syndrome is characterized by an acute and transient diastolic disfunction of the left ventricle, that simulates an acute coronary syndrome in its presentation, triggered by physical or psychological stress factors.

Methods. A series of patients with SCM seen in the Hospital Británico between the years 2011 and 2016 is shown. The characteristics of the studied group, and the associated clinical and epidemiological factors are described.

Results. Seventeen patients were included, with a mean age of 62.5 years, of which $94 \%$ were women. Over $70 \%$ had one or more cardiovascular risk factors. The majority of patients had elevated figures in blood of the myocardia biomarkers ultra-sensitive T Troponin and creatinphosphokynase. On sonography apical and mid-ventricular involvement of the left ventricle was observed, with moderate to severe effects on the left ventricular ejection fraction, and variable mitral incompetence.

Discussion: The incidence of this syndrome has increased over the past decade, due to a better understanding of the condition, and to the technological advances and availability of imaging methods. The findings of this series could help the rapid identification of patients with stress cardiomyopathy, and its clinical treatment

Keywords: takotsubo cardiomyopathy, stress cardiomyopathy, broken heart syndrome, apical ballooning syndrome.

\section{INTRODUCCIÓN}

La miocardiopatía por estrés (MCS) se define como un abombamiento apical agudo del ventrículo izquierdo (VI) de causa desconocida que asemeja una vasija de cuello angosto y fondo ancho o takotsubo, con resolución completa de la aquinesia apical en aproximadamente un mes (Figura 1). Las anormalidades de la contracción miocárdica típicamente ocurren en el VI; sin

I. Servicio de Clínica Médica.

2. Servicio de Cardiología,

3. Docencia e Investigación.

Hospital Británico de Buenos Aires

Correspondencia: Dr. Pablo Young. Servicio de Clínica Médica, Hospital Británico. Perdriel 74, CI280AEB CABA, Rep.Argentina. Email: pabloyoung2003@yahoo.com.ar

Los autores declaran no tener conflictos de intereses. Este estudio se realizó sin ninguna financiación.

Recibido: 03/05/2018 | Aceptado: 21/06/2018 embargo, han sido descripto casos que presentan compromiso biventricular ${ }^{1}$. Algunos pacientes pueden presentar alteraciones similares a la MCS, pero con una causa clínica conocida, por ejemplo, hemorragia subaracnoidea (HSA), taquicardia o feocromocitoma; estos cursan con un abombamiento apical secundario al aturdimiento miocárdico neurogénico ${ }^{2}$.

La incidencia de MCS es poco clara debido al subdiagnóstico y a la dificultad estadística asociada a la heterogeneidad de los estudios disponibles. Basados en reportes aislados, se ha estimado que este síndrome representa entre el 1.0-2.5\% de los pacientes con sospecha de SCA al ingreso hospitalario ${ }^{3}$. Posterior al año 2000, los reportes mundiales aumentaron rápidamente. En países occidentales, se ha calculado una incidencia de aproximadamente un 2-3\% ${ }^{4}$. Las mujeres mayores de 50 años constituyen el $80-100 \%$ de los casos en las diferentes series, las menores de 50 años solo representan el 3\% de los casos y los hombres entre el 4 y el $13 \% \%^{5-7}$.

Este síndrome se desencadena por estresores psicológicos o físicos. Dentro de los psicológicos se han reportado diversas situaciones (negativas o positivas), así como una gran 
variabilidad en la intensidad de estos gatillos ${ }^{8}$. También los desencadenantes físicos descriptos corresponden a diferentes etiologías tales como, esfuerzo físico extremo, patologías clínicas agudas, exacerbaciones de patologías crónicas, cirugías mayores, quimioterapia e inducción anestésica-19. La presentación clínica típica corresponde a la de un SCA, con dolor precordial anginoso, signosintomatología simpática, disnea de esfuerzo y cambios electro- cardiográficos de isquemia o injuria; también puede presentarse asintomático o con ángor atípico, disnea, síncope, palpitaciones, náuseas y/o emesis, mialgias, falla cardíaca aguda, arritmia ventricular, shock cardiogénico, paro cardiorrespiratorio e incluso muerte súbita ${ }^{1-52}$.

La clasificación acorde a la configuración imagenológica del VI se divide en formas típicas y atípicas, y por causas, en primarias y secundarias. Esta última clasificación, propuesta por el grupo de estudio español RETAKO, tendría implicancias pronósticas ${ }^{20}$.

Los estudios complementarios que contribuyen al diagnóstico consisten en el ECG, los biomarcadores cardíacos, la ecocardiografía y la cardiorresonancia $a^{3-5,20-23}$.

El manejo consiste en la monitorización hemodinámica y control de las complicaciones. Reportes de casos han demostrado que los $\beta$-bloqueantes y los inhibidores de la enzima convertidora de angiotensina (IECA) contribuyen al manejo del cuadro, aunque no generarían impacto en la recurrencia ${ }^{20-28}$.

Si bien el pronóstico es favorable en la mayor parte de los casos, se han reportado formas graves como la obstrucción del tracto de salida del VI, el shock cardiogénico o la ruptura de la pared libre del $\mathrm{VI}^{20,22,26,28}$. La mortalidad intrahospitalaria varía entre las series entre el 0-8\%

El objetivo principal del estudio consiste en describir las características clínicas de los pacientes con MCS registrados entre 2011 y 2016 en el Hospital Británico.

\section{MATERIALESY MÉTODOS}

\section{Diseño}

Estudio descriptivo, retrospectivo, transversal. La población a estudio comprende a los pacientes atendidos en la unidad de cuidados coronarios (UCO) del Hospital Británico de Buenos Aires. El trabajo fue aprobado por el Comité de Revisión Institucional del Hospital Británico de Buenos Aires.

\section{Recolección de variables}

Se realizó la búsqueda de eventos en la base de datos de epicrisis de la UCO del Hospital, en los campos correspondientes a diagnóstico, impresión diagnóstica o descripción de estudios por imágenes, con las palabras clave: takotsubo, miocardiopatía por estrés, acinesia apical con disfunción del ventrículo izquierdo y síndrome coronario agudo con cinecoronariografía sin lesiones; filtrándose los mismos con el uso de los criterios diagnósticos de MCS. Se analizaron los registros de los pacientes, obteniendo

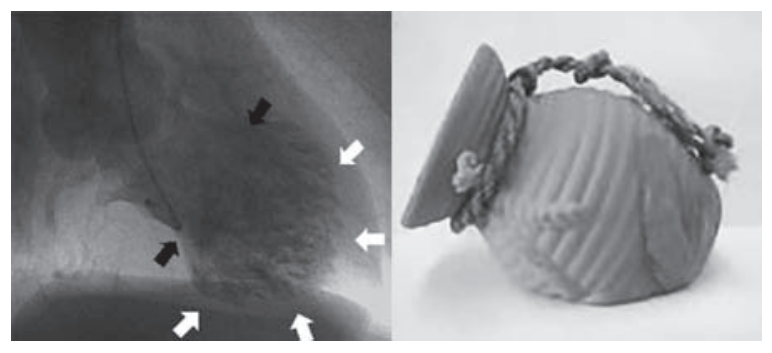

Figura I. Ventriculograma izquierdo durante la sístole. Las flechas negras señalan los segmentos basales con contractilidad conservada, en tanto que las flechas blancas apuntan a los segmentos medios y apicales aquinéticos. El conjunto asemeja un jarrón o takotsubo ${ }^{36,37}$.

datos demográficos, de presentación clínica, antecedentes patológicos y factores de riesgo coronario, además de datos electrocardiográficos, ecocardiográficos, bioquímicos e imagenológicos.

\section{Análisis estadístico}

Se realizó un análisis descriptivo. Las variables categóricas se expresaron en forma de frecuencias relativas, y las variables continuas como media y desvío estándar. Se utilizó el software GraphPadPrism 7.04.

\section{RESULTADOS}

Se encontraron 20 eventos de MCS, a los que se les aplicaron los criterios de la Mayo Clinic, definidos en la Tabla 1. Como resultado de la aplicación de estos criterios, se excluyeron 2 pacientes: uno por diagnóstico histopatológico de feocromocitoma y otro por presentar evidencia en el CCG de cardiopatía isquémica con stent (permeable) en el territorio de la arteria descendente anterior.

De los 18 eventos restantes, 2 pertenecían a una misma paciente que había presentado recurrencia, incluyendo de este modo, un total de 17 pacientes con 18 eventos cuyas características se detallan en la Tabla 2. Tal como se observa en dicha tabla, el $94.11 \%$ de los pacientes $(n=16)$ eran mujeres con una edad comprendida entre los 45 y 90 años, con el $23.5 \%$ menores de 50 años. Se encontraron factores estresantes en el $88.2 \%$ de los casos. El estresor psicológico representa el $60 \%$ y el físico el 40\% (clínico 70\% - quirúrgico 30\%).

Los pacientes requirieron en total una estancia hospitalaria de $4.1 \pm 3.5$ días, con un rango entre 1 y 14 días. Se observó que los 3 casos presentaron internaciones más prolongadas fueron, uno por accidente cerebrovascular (ACV) con múltiples intercurrencias, otra por sepsis a foco biliar y la última por enfermedad pulmonar obstructiva crónica (EPOC) reagudizada (Tabla 3).

Un $82.3 \%$ de los pacientes presentaba al menos un factor de riesgo cardiovascular (Figura 2A); de estos, 62.5\% correspondían a tabaquismo, $37.5 \%$ a hipertensión arterial (HTA), $37.5 \%$ a dislipemia (DLP) y $6.2 \%$ a obesidad. Como se observa en la Figura 2B, el 29.4\% de los pacientes presentaba antecedentes cardiológicos, $29.4 \%$ antecedentes de enfermedades crónicas y $64.7 \%$ ante- 
Tabla I. Criterios diagnósticos modificados de 2008 de la Mayo Clinic.

- Sospecha de infarto agudo de miocardio pordolor precordial y elevación del ST en el ECG en la fase aguda.

- Hipocinesia transitoria o acinesia de las regiones media y apical del VI e hipercontractilidad basal observada en la ventriculografía o ecocardiografía.

- Arterias coronarias normales confirmadas por arteriografía (obstrucción luminal $<50 \%$ en todas las arterias coronarias) en las primeras 24 horas del comienzo de los síntomas.

- Ausencia de traumatismo craneoencefálico significativo reciente, hemorragia intracraneal, sospecha de feocromocitoma, miocarditis o miocardiopatía hipertrófica.

cedentes neuropsiquiátricos. En cuanto a la presentación clínica, el ángor representa el 55.5\% de los casos (típico $70 \%$ y atípico $30 \%$ ), seguido de shock cardiogénico (16.7\%), síncope (11.1\%), disnea (11.1\%) y taquicardia ventricular o fibrilación ventricular (5.5\%) (Figura 2C). Hallazgos obtenidos en el ECG demostraron que el rango de la frecuencia cardíaca media resultó entre 60 y 120 lpm, las alteraciones más frecuentes fueron inversión de la onda T (58.8\%), supra-ST (17.6\%), infra-ST (17.6\%) y solo un 5.9\% (1 caso) tenía un ECG sin alteraciones isquémicas. El intervalo QT alargado se observó en un 52.9\% de los casos, el intervalo QT corto en un $11.78 \%$, las alteraciones de la repolarización precoz en un $11.76 \%$ y el bloqueo completo de rama derecha en un $5.9 \%$. De los sectores ventriculares comprometidos, la afectación más común fue la apical asociada a algunos segmentos mediales (64.7\%), seguida del apical puro (17.64\%), medioventricular puro (11.76\%) y global (5.88\%); además, un paciente presentaba compromiso biventricular. El deterioro de la fracción de eyección del VI (FEyVI) fue grave en el 43.8\% de los casos, moderada en el $12.5 \%$, leve en el $31.2 \%$ y sin compromiso (normal) en el $12.5 \%$.

El valor de creatinfosfokinasa (CPK) y troponina $\mathrm{T}$ ultrasensible (TTus) en promedio fue de $117.8 \pm 55.9 \mathrm{U} / \mathrm{l}$ y $227.4 \pm 242.2 \mathrm{ng} / \mathrm{l}$, respectivamente, con un rango entre $53 \mathrm{U} / \mathrm{l}$ a $240 \mathrm{U} / \mathrm{l}$ y $24 \mathrm{ng} / \mathrm{l}$ a $840 \mathrm{ng} / \mathrm{l}$, cada uno. De las troponinas, una paciente tenía troponina $\mathrm{T}$ sensible (TTs) de 0.03, los demás pacientes tenían TTus. A 2 pacientes se les realizó medición del propéptido natriurético cerebral N-terminal (NT-Pro-BNP) al ingreso, siendo marcadamente elevados (12000 pg/ml y 12100 $\mathrm{pg} / \mathrm{ml}$ ); sin embargo, no se realizó curva con esta prueba puesto que el seguimiento se hizo con clínica, ecocardiografía y niveles séricos de CPK y TTus.

A 5 pacientes se les realizó cardiorresonancia, que en ningún caso modificó el diagnóstico o tratamiento. De las CCG el $88.2 \%$ no presentaba lesiones, y del $11.8 \%$ restante ( 2 casos), uno correspondía a una lesión de cerca del $50 \%$ en la arteria marginal sin ruptura de placa ni trombosis y no concordante con el trastorno segmentario, y el otro a una lesión no significativa de la DA.

Respecto de las complicaciones, $29.4 \%$ presentaron insuficiencia mitral, pero ninguno evolucionó a formas graves como obstrucción del tracto de salida del VI ni perforación del septum interventricular. No se regis-
Tabla 2. Características de los pacientes internados con miocardiopatía por estrés entre 2011 y 2016.

\begin{tabular}{|l|c|}
\hline Sexo femenino (n; \%) & $18 ; 81 \%$ \\
\hline Edad (media \pm DE) & $60.7 \pm 12.7$ \\
\hline Días de internación (media \pm DE) & $4.1 \pm 3.5$ \\
\hline Pacientes con factor gatillo identificado (\%) & $88.2 \%$ \\
\hline Pacientes con insuficiencia mitral (\%) & $29.4 \%$ \\
\hline Pacientes con OTS del VI (\%) & $0 \%$ \\
\hline Pacientes fallecidos (\%) & $0 \%$ \\
\hline
\end{tabular}

DE: desvío estándar. OTS del VI: obstrucción del tracto de salida del VI.

Tabla 3. Comorbilidades asociadas en los pacientes internados con miocardiopatía por estrés entre 2011 y 2016.

\begin{tabular}{|c|c|c|}
\hline \multicolumn{3}{|l|}{ Antecedentes } \\
\hline $\begin{array}{l}\text { Patologías } \\
\text { cardiológicas } \\
(29.4 \%)\end{array}$ & $\begin{array}{l}\text { Patologías } \\
\text { neuropsiquiátricas } \\
(64.7 \%)\end{array}$ & $\begin{array}{l}\text { Otras patologías } \\
\text { crónicas } \\
(29.4 \%)\end{array}$ \\
\hline $\begin{array}{l}\text { Cardiopatía isquémica } \\
\text { en otro territorio } \\
\text { diferente de la DA. } \\
\text { (2 pacientes) }\end{array}$ & $\begin{array}{l}\text { Alzheimer + } \\
\text { epilepsia } \\
\text { (I paciente) }\end{array}$ & $\begin{array}{l}\text { Gammapatía de } \\
\text { significado incierto. } \\
\text { (I paciente) }\end{array}$ \\
\hline $\begin{array}{l}\text { Síncope de alto } \\
\text { riesgo cardiológico. } \\
\text { (I paciente) }\end{array}$ & $\begin{array}{l}\text { Síndrome vertiginoso } \\
\text { con traumatismo } \\
\text { craneoencefálico y } \\
\text { hemorragia } \\
\text { intracraneana con } \\
\text { colocación de } 3 \text { coils } \\
\text { I0 años previos al } \\
\text { episodio. } \\
\text { (I paciente) }\end{array}$ & $\begin{array}{l}\text { Hipotiroidismo } \\
\text { controlado. } \\
\text { (2 pacientes) }\end{array}$ \\
\hline $\begin{array}{l}\text { Fibrilación auricular. } \\
\text { (I paciente) }\end{array}$ & $\begin{array}{l}\text { Anorexia nerviosa } \\
\text { de difícil manejo + } \\
\text { trastorno bipolar + } \\
\text { trastorno de perso- } \\
\text { nalidad limítrofe. } \\
\text { (I paciente) }\end{array}$ & $\begin{array}{l}\text { Cáncer de mama. } \\
\text { (I paciente) }\end{array}$ \\
\hline $\begin{array}{l}\text { Estenosis displásica }+ \\
\text { taquicardia ventricular. } \\
\text { (I paciente) }\end{array}$ & $\begin{array}{l}\text { Trastorno depresivo. } \\
\text { (3 pacientes) }\end{array}$ & $\begin{array}{l}\text { Timoma. } \\
\text { (I paciente) }\end{array}$ \\
\hline $\begin{array}{l}\text { No se incluyen los } \\
\text { factores de riesgo } \\
\text { cardiovascular como } \\
\text { HTA en este apar- } \\
\text { tado }\end{array}$ & $\begin{array}{l}\text { Trastorno de ansie- } \\
\text { dad (asociado o no a } \\
\text { ataques de pánico) } \\
\text { (6 pacientes) }\end{array}$ & $\begin{array}{l}\text { EPOC severo. } \\
\text { (I paciente) }\end{array}$ \\
\hline
\end{tabular}

tró mortalidad asociada a la MCS ni por intercurrencias inmediatas. En 16 pacientes se indicó tratamiento con $\beta$-bloqueantes y IECA, y 1 solo con IECA por sus comorbilidades, todos con evolución favorable. Los pacientes que realizaron los controles posteriores $(7 \mathrm{pa}$ cientes) presentaron mejoría significativa, demostrándose por la normalización de los trastornos segmentarios y mejoría de los valores de la FEyVI, además de presentarse posteriormente asintomáticos para ángor y disnea; 10 pacientes no acudieron a los controles asignados o se negaron a realizarse estudios, por lo que perdieron seguimiento cardiológico y no se pudo documentar su evolución. Sin embargo, no se reportaron reinternaciones ni fallecimientos de causa cardiológica. Una paciente falleció 3 años después por enfermedad oncológica avanzada diagnosticada 2 años después de su internación en UCO. 


\section{DISCUSIÓN}

La MCS, también conocida como síndrome de acinesia apical transitoria, miocardiopatía de takotsubo (por la similitud con una trampa para pulpos japonesa) (Figura 1) , fue descripta en 1990 por Sato et al., y su incidencia ha aumentado a lo largo de los años $5,20,22,29$ 34,53. Se han propuesto varios criterios diagnósticos, siendo los de la Mayo Clinic, de 2008, los más usados ${ }^{20-23}$.

Se ha postulado que el estrés, psicológico o físico, se relacionaría con múltiples mecanismos como vasoespasmo coronario, estrés oxidativo, desórdenes microcirculatorios, predisposición genética, obstrucción del tracto de salida, déficit estrogénico e hiperexcitación simpática. Más aún, se postula que esta es inducida comúnmente donde hay disfunciones cardíacas similares en pacientes con feocromocitoma y casos de desarrollo de MCS secundario a la administración de epinefrina y dobutamina $a^{22,23,39-41-43}$.

Se han descripto como factores desencadenantes, factores emocionales (happy and broken heart syndrome) o físicos (esfuerzos, traumatismos, patologías agudas cardíacas y no cardíacas, infecciones, cirugías, inducción anestésica, uso de inotrópicos, intoxicaciones y quimioterapia $^{9-19}$.

La presentación clínica característica corresponde a la de un SCA, con dolor precordial anginoso, signosintomatología simpática, disnea de esfuerzo y cambios electrocardiográficos de isquemia o injuria. La diferencia inicial entre la MCS y el SCA es a menudo complicada debido a su presentación, sumada a que en este grupo etario pueden encontrarse lesiones no significativas en la CCG. Existen distintas clasificaciones de la MCS, según los criterios utilizados ${ }^{22}$. En cuanto a características imagenológicas, se dividen en formas típicas, que representan el 66\% de los casos y comprometen el ápex y algunos segmentos medioventriculares con hipercontractilidad basal compensadora, y formas atípicas medioventriculares aisladas, focales o basales, y compromiso del ventrículo derecho.

En cuanto a las causas, se dividen en primarias (sin causa evidente, generalmente producidas por estrés psicológico extremo) y secundarias (pacientes con enfermedades concomitantes graves, generalmente el $20 \%$ de los casos), que tienen peor pronóstico ${ }^{20}$.

En el ECG son frecuentes los cambios en la repolarización, los supradesniveles del segmento ST, evolucionan a inversiones de la onda $T$ progresivas, también hay alargamiento del intervalo QT. Estas anomalías mejoran con el tiempo y es rara la presencia de las ondas $\mathrm{Q}$ patológicas y aún más su persistencia ${ }^{3,22,46-49}$.

Los biomarcadores actuales no son específicos para diferenciar entre los SCA y la miocardiopatía por estrés ${ }^{3,22,47}$. Se ha descripto que por lo general la elevación de las TTus y la CPK son menos llamativas que en el IAMCEST ${ }^{50-52}$. El NT-Pro-BNP, en comparación, suele tener un pico más ele-

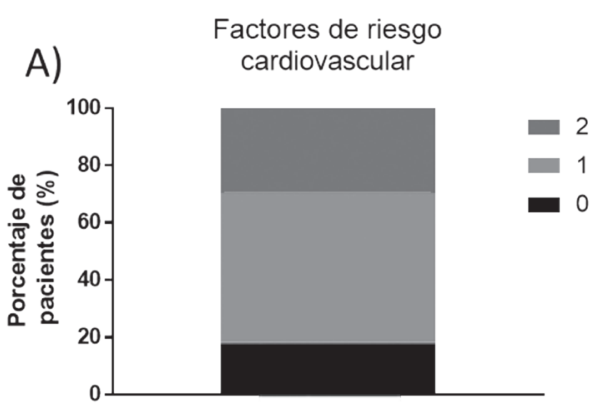

B)

Antecedentes

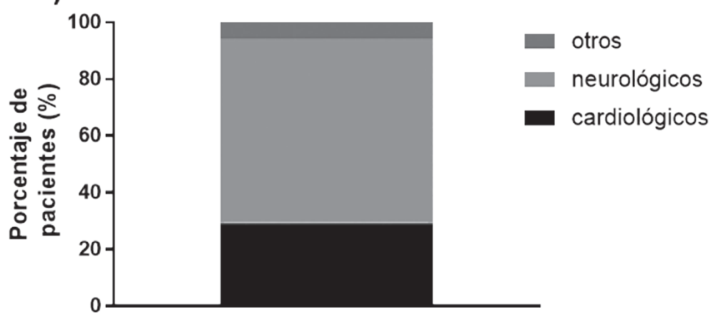

C) Formas de presentación

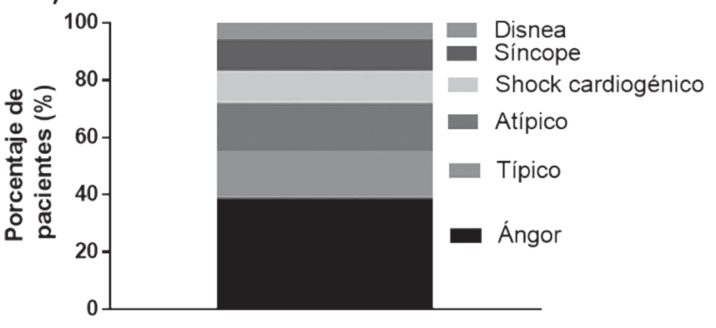

Figura 2. Descripción de los antecedentes la población estudiada.

vado que en el SCA, y está relacionado directamente con el grado de disfunción del VI.

En cuanto a estudios de imagen que contribuyen al diagnóstico, la ecocardiografía Doppler es el estudio de elección que descarta complicaciones, muestra anomalías segmentarias y valora la fracción de eyección. Además, tiene gran utilidad para el seguimiento y pronóstico ${ }^{22,47}$. La cinecoronariografía excluye lesiones obstructivas de las arterias coronarias y se puede realizar tratamiento por angioplastia primaria en caso de presentarlas ${ }^{3,22,47}$. La cardiorresonancia es útil para descartar otras miocardiopatías como diagnóstico diferencial. La biopsia del miocardio no es necesaria para el diagnóstico y no tiene un patrón específico, presenta aumento del tejido conectivo y lesiones focales reversibles de miocitolisis con infiltración mononuclear y fibrosis ${ }^{3,22}$.

La evolución y pronóstico son benignos generalmente, en especial al compararlos con los SCA, dado a que se trata de una disfunción del VI que se recupera ad integrum en un tiempo no mayor a 3 semanas en la mayor parte de los casos ${ }^{1-6,20-38,54,55}$. No obstante, no está exenta de complicaciones agudas, las cuales ocurren en un $18,9 \%$ de los $\operatorname{casos}^{6,30,39}$, como arritmias, insuficiencia mitral, falla cardíaca aguda, shock 
cardiogénico y otras más infrecuentes como neumotórax, pericarditis, ruptura de la pared libre del VI, perforación del septum interventricular o de los músculos papilares, trombos intracavitarios y fenómenos tromboembólicos ${ }^{3,6,20,22,38,53-56}$. El monitoreo de la evolución clínica es esencial para prevenir o tratar estas posibles complicaciones agudas. La mortalidad varía entre un $0-12 \%$, la mortalidad intrahospitalaria se ha estimado en $1 \%$, y al año, en $2 \%{ }^{6,23,30}$. La mortalidad y la morbilidad de los pacientes es más baja que en el SCA, y la función ventricular podría determinar el pronóstico. La recurrencia es rara y se ha descripto alrededor del 3\% ${ }^{6,23,29,30,38,53-56}$.

Los pacientes estudiados en esta serie se han caracterizado por ser, en casi su totalidad, mujeres con edades por encima de los 50 años y al menos 1 factor de riesgo cardiovascular asociado a patología crónica y/o patología neuropsiquiátrica (generalmente trastorno de ansiedad). Nos fue posible identificar los gatillos en la mayor parte de los pacientes, prevaleciendo los psicológicos sobre los físicos. El desencadenante común fue un estresor emocional negativo, similar a lo comunicado en la literatura mundial. Aquellos con detonante físico se presentaron con formas más graves y requirieron más días de internación que los pacientes con estresores emocionales. En nuestra serie predominó la afección apical asociada a algunos segmentos mediales (64.7\%) y en cuanto al ECG los hallazgos más frecuentes fueron la inversión de la onda $\mathrm{T}$ (58.8\%) y el alargamiento del intervalo QT (52.9\%), similar a lo antes publicado. Los marcadores bioquímicos presentaron niveles más altos que los descriptos en la literatura, en forma similar a la serie del RETAKO, que reportó niveles de CPK de 200 [126-336] $]^{22}$. A dos pacientes se les realizó medición de NT-Pro-BNP al ingreso, que fueron marcadamente elevados (12 000 y 12 100); sin embargo, no se les hizo seguimiento con esta prueba dada la evidente mejoría clínica. En nuestra experiencia, la cardiorresonancia no cambió conducta en los casos en los que se realizó.

Se encontraron resultados similares a los informados previamente en diferentes publicaciones en cuanto a la caracterización de los pacientes: el perfil fue el de una paciente mayor de 50 años, cuya presentación al ingreso fue con dolor precordial anginoso típico, asociado a inversión de la onda $\mathrm{T}$ y alargamiento del segmento QT en el ECG, elevación de los biomarcadores cardíacos, sin alteraciones en la CCG, con antecedentes neuropsiquiátricos, por lo menos 1 factor de riesgo cardiovascular, asociado a patologías crónicas y con un detonante psicológico negativo, presentando en la ecocardiografía Doppler un compromiso apical y medioventricular con afectación de FeVI en grado moderado a severo e insuficiencia mitral variable, con una estadía hospitalaria de aproximadamente 6 días y evolución posterior favorable. El pronóstico fue favorable y al igual que en la literatura mundial, no tuvimos casos fatales. Por otro lado, una paciente experimentó recurrencia del cuadro en el período estudiado, diferente a lo publicado.

Informar las características de esta serie de pacientes podría contribuir a comprender la mejor caracterización de esta entidad en nuestra comunidad. Sin embargo, todavía quedan algunos interrogantes respecto a su fisiopatología, factores de riesgo, manejo óptimo, seguimiento, evolución a largo plazo y prevención, para lo cual todavía falta pasar la prueba del tiempo con estudios prospectivos y de seguimiento de estos pacientes.

\section{BIBLIOGRAFÍA}

I. Daoko J, Rajachandran M, Savarese R, Orme J. Biventricular takotsubo cardiomyopathy case study and review of literature. Tex Heart Inst J 2013;40:305-I I.

2. Ancona F, Bertoldi LF, Ruggieri F, et al. Takotsubo cardiomyopathy and neurogenic stunned myocardium: similar albeit different. Eur Heart J 201 6;37:2830-2.

3. Roshanzamir S, Showkathali R.Takotsubo cardiomyopathy a short review. Curr Cardiol Rev 20 13;9:191-6.

4. Andrade AA, Stainback RF.Takotsubo Cardiomyopathy.Tex Heart Inst J 201 4:41:299-303.

5. Scantlebury DC, Prasad A. Diagnosis of Takotsubo cardiomyopathy - Mayo Clinic Criteria-. Circ J 2014;78:2 129-39.

6. Prasad A, Lerman A, Rihal CS. Apical ballooning syndrome (TakoTsubo or stress cardiomyopathy): A mimic of acute myocardial infarction. Am Heart J 2008; I 55:408- 17.

7. Kurisu S, Kihara Y. Clinical management of takotsubo cardiomyopathy. Circ J 20I4;78:I559-66.

8. Sharkey SW, Maron BJ. Epidemiology and clinical profile of takotsubo cardiomyopathy. Circ J 2014;78:21 19-28.

9. De Giorgi A, Fabbian F, Parisi C, et al. Takotsubo cardiomyopathy

and acute infectious diseases: a mini-review of case reports. Angiology 20 I 5;66:257-6 I.

10. Galea F,Abela GP, Felice H.Takotsubo cardiomyopathy in chronic infection. Scott Med J 20 I3;58:el I -4.

I I. De Giorgi A, Fabbian F,Tiseo R, et al.Takotsubo cardiomyopathy and endocrine disorders: a mini-review of case reports. Am J Emerg Med 20 | 4;32:14 | 3-7.

12. Chiariello GA, Bruno P, Colizzi C, Crea F, Massetti M. Takotsubo Cardiomyopathy Following Cardiac Surgery. J Card Surg 2016;31:89-95.

13. Hammer N, Kühne C, Meixensberger J,Hänsel B,Winkler D.Takotsubo cardiomyopathy - An unexpected complication in spine surgery. Int J Surg Case Rep 20 15;6:172-4.

14. Bhojraj S, Sheth S, Pahlajani D. Postoperative Takotsubo cardiomyopathy. Ann Card Anaesth 20 I4; 17:157-60.

15. Goel S, Sharma A, Garg A, Chandra A, Shetty V.Chemotherapy induced takotsubo cardiomyopathy. World J Clin Cases 20| 4;2:565-8

16. Ovadia D, Esquenazi Y, Bucay M, Bachier CR. Association between 
takotsubo cardiomyopathy and axitinib: case report and review of the literature. I Clin Oncol 2015;33:el-3.

17. Nogi M, Fergusson D, Chiaco JM. Mid-ventricular variant takotsubo cardiomyopathy associated with cannabinoid hyperemesis syndrome: a case report. Hawaii J Med Public Health 20 |4;73: I I 5-8.

18. Mazzola P,Picone D,Anzuini A, Corsi M, Bellelli G,Annoni G.Takotsubo cardiomyopathy in an 81-year-old woman after injection of bone cement during hemiarthroplasty:An orthogeriatric case report. Intl | Surg Case Rep 20 |6; | 8:37-4|

19. Eliades M, El-Maouche D, Choudhary C, Zinsmeister B, Burman KD. Takotsubo cardiomyopathy associated with thyrotoxicosis: a case report and review of the literature. Thyroid 20 14;24:383-9.

20. Nuñez-Gil I],Mejía-Rentería HD,Martínez-Losas P.Actualización práctica en síndrome de Takotsubo. Med clin (Barc) 2016:146:2 12 -7.

21. Veillet-Chowdhury M, Hassan SF,Stergiopoulos K.Takotsubo cardiomyopathy: a review. Acute Card Care 20 14;16:15-22.

22. Ono R, Falcão LM. Takotsubo cardiomyopathy systematic review: Pathophysiologic process, clinical presentation and diagnostic approach to Takotsubo cardiomyopathy. Int J Cardiol 20 I 6;209: I96-205.

23. Castillo Rivera AM, Ruiz-Bailén M, Rucabado Aguilar L.Takotsubo cardiomyopathy--a clinical review. Med Sci Monit 20 I I; $17:$ RA I 35-47

24. Isogai T,Matsui $H$,Tanaka $H$, Fushimi K, Yasunaga $H$. Early beta-blocker use and in-hospital mortality in patients with Takotsubo cardiomyopathy. Heart 2016; 102:1029-35.

25. Lyon AR, Bossone E, Schneider B, Sechtem U, Citro R, Underwood $\mathrm{SR}$, et al. Current state of knowledge on Takotsubo syndrome: A Position Statement from the Taskforce on Takotsubo Syndrome of the Heart Failure Association of the European Society of Cardiology. Eur J Heart Fail 20 16; 1 8:8-27.

26. Templin C, Ghadri JR, Diekmann J, et al. Clinical Features and Outcomes of Takotsubo (Stress) Cardiomyopathy. N Engl J Med 2015;373:929-38

27. Ghadri IR, Ruschitzka F, LüscherTF,Templin C. Takotsubo cardiomyopathy: still much more to learn. Heart 20 14; 100: I 804- 12.

28. Komamura K, Fukui M, Iwasaku T, Hirotani S, Masuyama T. Takotsubo cardiomyopathy: Pathophysiology, diagnosis and treatment. World J Cardiol 20 14;6:602-9.

29. Akashi YJ, Goldstein DS, Barbaro G, Ueyama T. Takotsubo cardiomyopathy: a new form of acute, reversible heart failure. Circulation 2008; I | 8:2754-62

30. Tsuchihashi K, Ueshima K, UchidaT, Oh-mura N, Kimura K, Owa M, et al.Transient left ventricular apical ballooning without coronary artery stenosis: a novel heart syndrome mimicking acute myocardial infarction. Angina Pectoris-Myocardial Infarction Investigations in Japan. J Am Coll Cardiol 200 I;38: I I-8.

31. Abe Y, Kondo M, Matsuoka R, Araki M, Dohyama K, Tanio H. Assessment of Clinical Features in Transient Left Ventricular Apical Ballooning. J Am Coll Cardiol 2003;41:737-42.

32. Lyon AR, Rees P, Prasad S, Poole-Wilson PA, Harding SE. Stress (Takotsubo) cardiomyopathy - a novel pathophysiological hypothesis to explain catecholamine-induced acute myocardial stunning. Nature Clin Pract Cardiovasc Med 2008;5:22-9

33. Kawaj T, Shiomi H, Morimoto T, Tazak J, Imai M, Saito N, et al. Clinical impact of left ventricular outflow tract obstruction in takotsubo cardiomyopathy. Circ J 20 I 5; 79:839-46.

34. Yoshikawa T. Takotsubo cardiomyopathy, a new concept of cardiomyopathy: clinical features and pathophysiology. Int J Cardiol 2015; | 82:297-303

35. Rawasia WF, Pachika A, Ikram S. Diagnostic dilemma:Takotsubo cardiomyopathy versus acute coronary syndrome. I Invasive Cardiol 2014;26:E82-4.

36. Finn B, Young P, Bruetman J. Takotsubo, discinesia apical transito- ria. Presentación de 4 casos y revisión de la literatura. Medicina (B Aires) 2005;65:4I5-8.

37. Vadalá S,Pellegrini D,Verdaguer MF,Schrappe M,Álvarez J,Bruetman JE. Cardiomiopatía inducida por estrés (Takotsubo) en una paciente con anorexia nerviosa. Medicina (B Aires) 2014;74:222-4.

38. Rau CM, Kauffmann M, Rau CL, Cereceda M, Castro G, Massardo T. Síndrome deTako-tsubo: caso clínico. Rev Med Chile 20 I 1; 139:348-52.

39. Fortuna L, Moreyra E, Hamity L, et al. Síndrome de Takotsubo, experiencia en tres instituciones de la ciudad de Córdoba. Medicina (B Aires) 2014;74:42-8.

40. Coupez E, Eschalier R, Pereira B, et al. A single pathophysiological pathway in Takotsubo cardiomyopathy: Catecholaminergic stress. Arch Cardiovasc Dis 2014;107:245-52.

41. Y-Hassan S. Divergence in the results of plasma catecholamine levels in different studies on patients with takotsubo syndrome: Why? J Cardiol 2016;68:89.

42. Wittstein IS, Thiemann DR, Lima JA, et al. Neurohumoral features of myocardial stunning due to sudden emotional stress. N Engl ] Med 2005;352:539-48.

43. Chou AY, Saw J. Basis for sex-specific expression of Takotsubo cardiomyopathy, cardiac syndrome $X$, and spontaneous coronary artery dissection. Can J Cardiol 2014;30:738-46

44. Draca S. A possible relationship between Takotsubo cardiomyopathy and female sex steroid-related modulation of functional cerebral asymmetry. Med Hypotheses 2015;84:238-40.

45. Khalid N. Microcirculatory disorder hypothesis in Takotsubo cardiomyopathy. Int J Cardiol 20 15;195:29.

46. Ghadri JR, Sarcon A, Diekmann J, et al. Happy heart syndrome: role of positive emotional stress in takotsubo syndrome. Eur Heart I 201 6;37:2823-9.

47. Bossone E, Lyon A, Citro R, et al. Takotsubo cardiomyopathy: an integrated multi-imaging approach. Eur Heart J Cardiovasc Imaging 2014;15:366-77.

48. Shimuzu M, Nishizaki M,Yamawake N, et al. J wave and fragmented QRS formation during the hiperacute phase in Takotsubo cardiomyopathy. Circ J 2014;78:943-9.

49. Sinha A, Rassiwala J, Goldschlager N. Takotsubo cardiomyopathy: how T waves behave under stress. JAMA Intern Med 2015; 175:842-4

50. Mugnai G,Vassanelli F,Pasqualin G, et al. Dynamic changes of repolarization abnormalities in takotsubo cardiomyopathy. Acta Cardiol 2015;70:225-32.

5।. Novo G, Giambanco S, Bonomo V, et al. Troponin I/ejection fraction ratio: a new index to differentiate Takotsubo cardiomyopathy from myocardial infarction. Int J Cardiol 20।5; 180:255-7.

52. Doyen D, Moceri P, Chiche $O$, et al. Cardiac biomarkers in Takotsubo cardiomyopathy. Int J Cardiol20 |4; |74:798-80 I.

53. Sato H,Tateishi H, Uchida T, et al.Takotsubo type cardiomyopathy due to multivessel spasm. In: Kodama K, Haze K, Hon M, eds. Clinical aspect of myocardial injury: from ischemia to heart failure [in Japanese] Tokyo: Kagakuhyouronsya Co, 1990; 56-64.

54. De Backer O, Debonnaire P, Gevaert S, Missault L, Gheeraert P, Muyldermans L. Prevalence, associated factors and management implications of left ventricular outflow tract obstruction in takotsubo cardiomyopathy: a two-year, two-center experience. BMC Cardiovasc Disord 2014; |4:147.

55. Singh K, Carson K, Hibbert B, Le May M. Natural history of cardiac arrest in patients with takotsubo cardiomyopathy. Am J Cardiol 2015; I 15:1466-72.

56. Singh K, Carson K, Usmani Z, Sawhney G, Shah R, Horowitz J. Systematic review and meta-analysis of incidence and correlates of recurrence of takotsubo cardiomyopathy. Int J Cardiol 20 I 4; 174:696-70 I. 